\title{
Correction: Loeber, Gerard. ISNS 9th International Symposium, The Hague, The Netherlands, September 11-14, 2016. Int. J. Neonatal Screen. 2016, 2, 5
}

\section{Gerard Loeber}

ISNS Office, 3721 CK Bilthoven, The Netherlands; gerard.loeber@gmail.com

Academic Editor: Ralph Fingerhut

Received: 20 October 2016; Accepted: 20 October 2016; Published: 21 April 2017

The author wishes to make the following correction to their paper published in the International Journal of Neonatal Screening [1].

1. The affiliation of Dr. Kate Hall in the I23 Part as shown in the Appendix should read "West Midlands, UK".

2. The affiliation of Dr. Martina C. Cornel in the O23 Part should read "VU University Medical Center, Section Community Genetics, Department of Clinical Genetics and EMGO Institute, Amsterdam, The Netherlands".

3. In P49, the second sentence in the Results Section should be changed to "Parents with a low tolerance for ambiguity were favorable toward this type of screening".

4. The conclusion in P30 needs to be replaced with the following: Conclusion: An additional 12 babies with confirmed CF were detected following second IRT testing and will benefit from early intervention and follow-up. This provides supporting evidence for the continuation of this component of the screening protocol.

5. In O06, the author would like to replace the word "article" with "review" in the second paragraph.

6. In P52, the authors would like to change all the affiliations to the following:

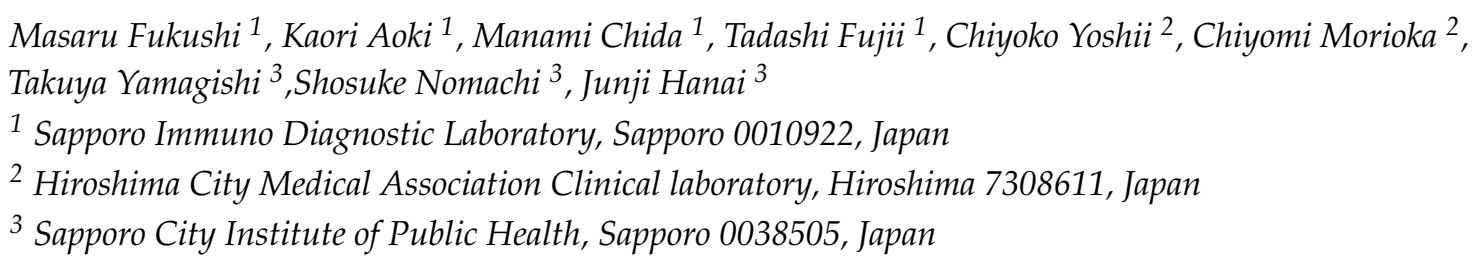

7. In P73, the authors would like to replace the affiliations with the following:

Felix Votava ${ }^{1}$, Viktor Kožich ${ }^{2}$, Petr Chrastina ${ }^{2}$, Karolina Pešková ${ }^{2}$, Tomáš Adam $^{3}$, David Friedecký ${ }^{3}$, Eva Hlídková ${ }^{3}$, Hana Vinohradská ${ }^{4}$, Petr Dejmek ${ }^{1}$, Veronika Krulišová ${ }^{5}$, Andrea Holubová ${ }^{5}$, Jan David ${ }^{1}$, Milan Macek Jr ${ }^{5}$, Renata Gaillyová ${ }^{6}$, Iveta Valášková ${ }^{6}$

${ }^{1}$ Department of Pediatrics, 3rd Faculty of Medicine, Charles University Prague and University Hospital Kralovske Vinohrady Prague, Czech Republic

${ }^{2}$ Institute of Inherited Metabolic Disorders, 1st Faculty of Medicine, Charles University and General University Hospital Prague, Czech Republic

${ }^{3}$ Laboratory for Inherited Metabolic Disorders, Faculty of Medicine and Dentistry Palacky University and University Hospital Olomouc, Czech Republic

${ }^{4}$ Department of Clinical Biochemistry, Faculty of Medicine Masaryk University and University Hospital Brno, Czech Republic 
${ }^{5}$ Department of Biology and Medical Genetics, 2nd Faculty of Medicine, Charles University and Motol University Hospital Prague, Czech Republic

${ }^{6}$ Department of Medical Genetics, Faculty of Medicine Masaryk University and University Hospital Brno, Czech Republic

8. In P20, the authors would like to change the affiliation: "Cytogenomic Medical Laboratory, Newborn Screening, Bucharest, Romania". In the second paragraph, first line, remove the sign ";" after the name Alfred Rusescu. In the third paragraph, line 5, remove "ornithine" (it should be phenylalanine, leucine+isoleucine, glycine, glutamic and aspartic acids). In addition, Ramona Mohora and Sivia Maria Stoicescu would like to change their affiliations: Carol Davila University of Medicine and Pharmacy, Bucharest, Romania.

9. In P28, the authors would like to change the title to: "Very High Incidence of Low Vitamin B12 in Estonian Newborns" replacing "Incidents" by "Incidence".

10. In O17, the authors would like to make the following changes to the affiliations:

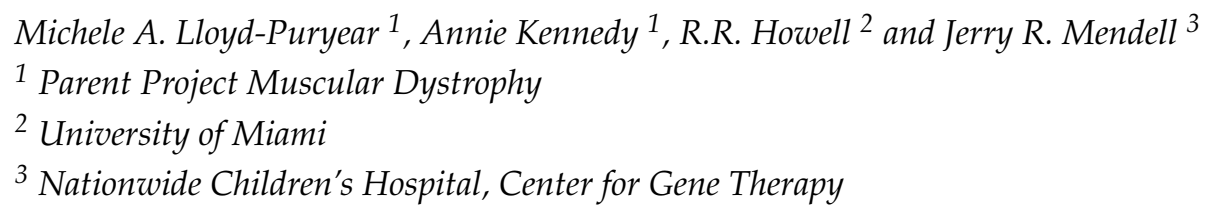

11. In O02, the author would like to change DBS" into "DBS'" in the sentence "Therefore, we now developed a non-destructive method which allows to predict a DBS" Hct using non-contact diffuse reflectance spectroscopy".

12. In P50, the list of authors should be replaced with: Veronica Wiley, Rosie Junek, Crystyna Smith, Tiffany Wotton, Won Tae Kim and Jake Berry

We would like to apologize for any inconvenience caused to the readers by these changes. The changes do not affect the scientific results. The manuscript will be updated and the original will remain available on the article webpage.

\section{Reference}

1. Loeber, G. ISNS 9th International Symposium, The Hague, The Netherlands, September 11-14, 2016. Int. J. Neonatal Screen. 2016, 2, 5. [CrossRef]

(C) 2017 by the author. Licensee MDPI, Basel, Switzerland. This article is an open access article distributed under the terms and conditions of the Creative Commons Attribution (CC BY) license (http:/ / creativecommons.org/licenses/by/4.0/). 